\title{
A Fused Ring Electron Acceptor with Decacyclic Core Enables over 13.5\% Efficiency for Organic Solar Cells
}

\author{
Dan He, Fuwen Zhao, * Jingming Xin, Jeromy James Rech, Zhixiang Wei, * Wei Ma, \\ Wei You, Bao Li, Li Jiang, Yongfang Li, and Chunru Wang*
}

Extending $\pi$-conjugation of donor units in fused ring electron acceptors (FREAs) promises to reinforce intramolecular charge transfer for smaller bandgaps, and enhances intermolecular interactions for higher charge mobility. Despite these advantages, power conversion efficiencies (PCEs) of FREAs with a large planar donor core are still below $12 \%$. Herein, a fused decacyclic donor unit, IDC, is developed, and the FREA, IDCIC, whose optical bandgap is $1.45 \mathrm{eV}$, is synthesized. The FTAZ:IDCIC-based organic solar cell (OSC) with a binary additive, chloronaphthalene and 1,8-diiodooctane (CN\&DIO), affords a remarkable PCE of $13.58 \%$, which is among the highest efficiencies of OSCs. The binary additive plays a crucial role in the morphology of FTAZ:IDCICbased OSCs. The addition of DIO promotes IDCIC to aggregate and enhances domain size and domain purity in FTAZ:IDCIC blend film for its poor solubility in DIO, while the addition of chloronaphthalene (CN) inhibits the aggregation, facilitates IDCIC to diffuse into the donor, and reduces domain size and domain purity for its excellent solubility in $\mathrm{CN}$. The binary additive, CN\&DIO, compromises the effect of the two; thus FTAZ:IDCIC-based OSCs with $0.25 \%$ CN\&DIO obtain moderate domain size and high domain purity simultaneously, achieving the least charge recombination for the highest $I_{\mathrm{SC}}$ $\left(21.98 \mathrm{~mA} \mathrm{~cm}^{-2}\right.$ ) and fill factor (FF) (71.03\%).
Bulk-heterojunction organic solar cells (OSCs) have been subjected to increasing interest due to their advantages of easy fabrication, low-cost, light-weight, mechanical flexibility, etc. ${ }^{[1]}$ Extensive efforts have been made in materials and physical fundamental to develop highperformance OSCs over the last two decades. ${ }^{[2]}$ The active layer materials, namely donor (D) and acceptor (A), are crucial in the development of OSC, since their photovoltaic properties determine the limit on the maximum output of the devices. ${ }^{[3]}$ Recently, nonfullerene acceptors have attracted much attention owing to their merits: i) strong absorption in visible or near infrared ray region to harvest sunlight efficiently and promise high short-circuit current density $\left(U_{\text {sc }}\right)$, ii) good energy level tunability to achieve high open-circuit voltage $\left(V_{\mathrm{OC}}\right)$ and efficient exciton dissociation simultaneously, and iii) diverse and facile chemical modification to control the structural properties, compared with traditional fullerene acceptors. ${ }^{[4]}$ Among them,

Dr. D. He, Dr. F. Zhao, Dr. L. Jiang, Prof. Y. Li, Prof. C. Wang Institute of Chemistry

Chinese Academy of Sciences

Beijing 100190, China

E-mail: zhaofuwen@iccas.ac.cn; crwang@iccas.ac.cn

J. Xin, Prof. W. Ma

State Key Laboratory for Mechanical Behavior of Materials

Xi'an Jiaotong University

Xi'an 710049, China

J. J. Rech, Prof. W. You

Department of Chemistry

University of North Carolina at Chapel Hill

Chapel Hill, NC 27599-3290, USA

Prof. Z. Wei

Key Laboratory of Nanosystem and Hierarchical Fabrication

National Center for Nanoscience and Technology

Beijing 100190, China

E-mail:weizx@nanoctr.cn

Dr. B. Li

School of Chemistry and Chemical Engineering

Henan Normal University

Xinxiang 453007, China

The ORCID identification number(s) for the author(s) of this article can be found under https://doi.org/10.1002/aenm.201802050.

DOI: 10.1002/aenm.201802050 fused ring electron acceptors (FREAs) exhibit outstanding photovoltaic performance. FREA generally contains a fused ring donor unit with side chains attached to the bridging-carbon atom and hanged out of the molecular plane in the center, and two compact strong electron-withdrawing structures at both ends. The FREA possesses three traits as follows: 1) the fused ring donor unit affords a planar conjugated center to facilitate molecule packing and can effectively tune energy levels and absorption; 2) the side chains can not only ensure sufficient solubility for solution process, but also inhibit excessive aggregation for fine morphology; 3) the electron-withdrawing structure at the end can bring about intense intramolecular charge transfer for small bandgap and induce strong intermolecular interaction for $J$-aggregation and high electron mobility. ${ }^{[5]} \mathrm{As}$ a result, numerous high-efficient FREAs have been developed and the power conversion efficiencies (PCEs) of the OSCs based on them have increased to over $14 \%$ recently. ${ }^{[6]}$

As discussed above, the fused ring donor unit in the center has an important influence on the electronic structure and intermolecular interaction of FREAs, thus impacting their photovoltaic performance. Most reported FREAs are based on rather simple fused aromatics, such as fluorene, ${ }^{[7]}$ indacenodithiophene, ${ }^{[8]}$ naphthalene(cyclopentadithiophene), ${ }^{[9]}$ 


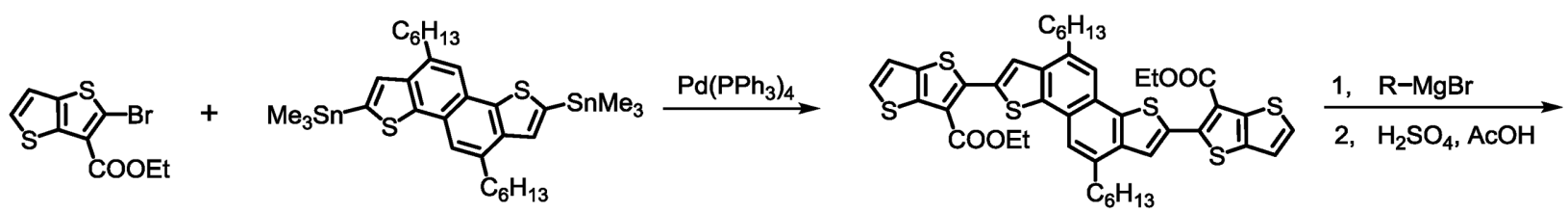

1

2

3
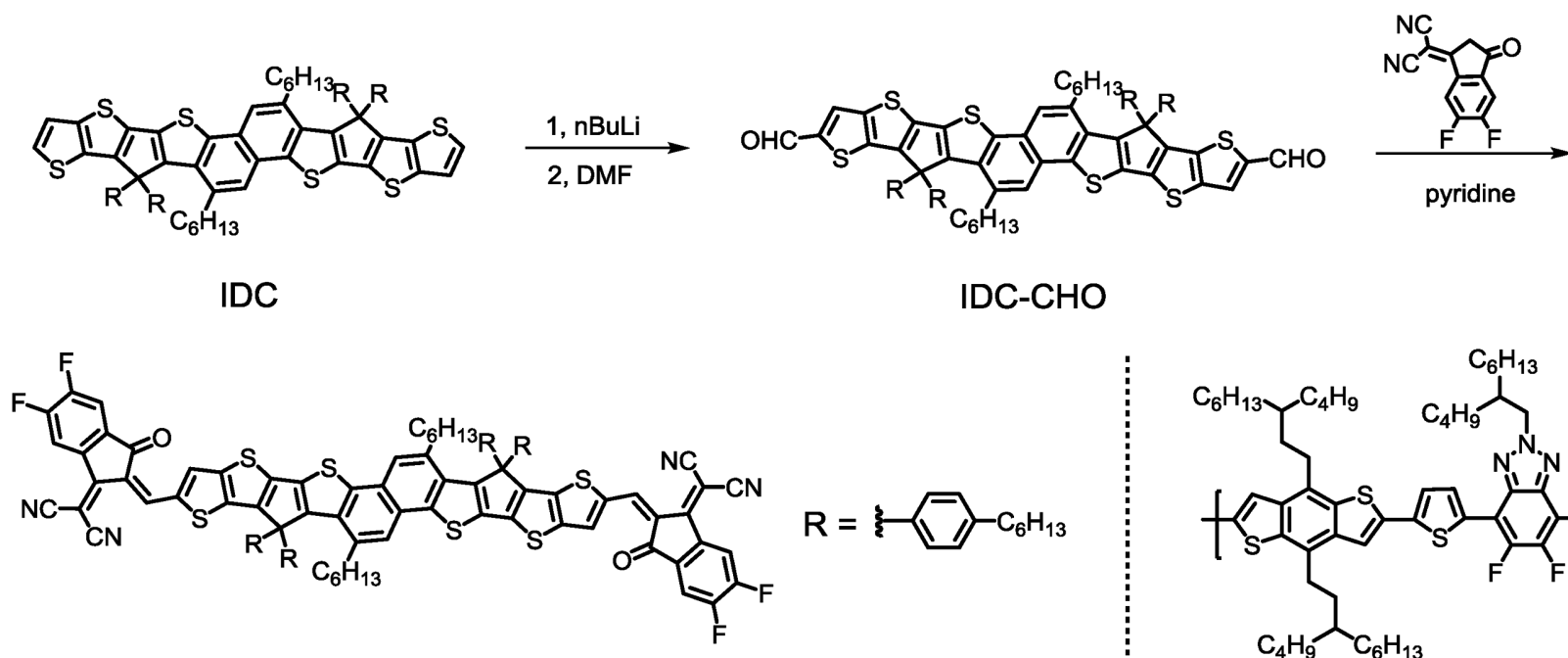

IDCIC

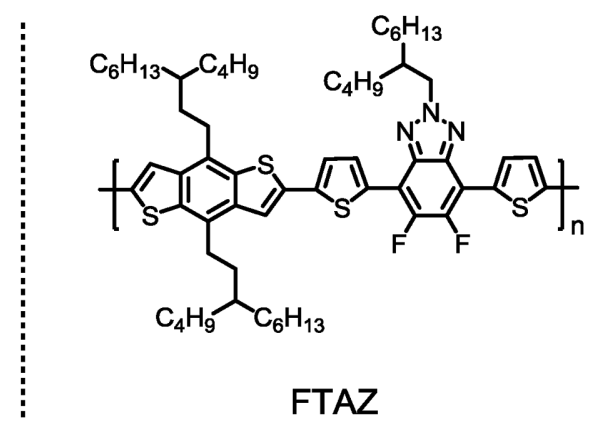

Scheme 1. Synthetic route of IDCIC and chemical structure of the donor FTAZ.

indacenodithieno[3,2-b]thiophene, ${ }^{[6 a, 10]}$ and benzodi(cyclopentadithiophene).$^{[11]}$ However, these units suffer relatively weak electron-donating capability and intermolecular interaction, leading to relatively large bandgap $(\geqq 1.5 \mathrm{eV})$ and low electron mobility $\left(10^{-5}-10^{-4} \mathrm{~cm}^{2} \mathrm{~V}^{-1} \mathrm{~s}^{-1}\right)$ for FREAs. It has been well established that extending the $\pi$-conjugation of fused ring donor unit in the FREA would improve its electron-donating capacity to reinforce the intramolecular charge transfer effect for smaller bandgap and enhance intermolecular interaction to facilitate molecular packing for higher charge mobility. ${ }^{[5,12]}$ Therefore, it is necessary to develop FREAs with larger fused ring donor cores to further improve the PCE. Up to date, a few large fused ring donor units have been developed and used as building blocks to prepare FREAs, but most of OSCs based on them still suffer relatively low PCE (within 12\%). ${ }^{[13]}$ Motivated by these, we developed a fused decacyclic donor unit via fusing naphtho[1,2-b:5,6- $b$ ] dithiophene with two thieno[3,2-b]thiophene and synthesized the FREA, IDCIC, (Scheme 1). The synthesis, thermal stability, optical and electrochemical properties, and photovoltaic performance of IDCIC are discussed below. In addition, the effect of additive on the morphology and photovoltaic performance of FTAZ:IDCIC based OSCs is also investigated. The FTAZ:IDCIC based OSCs with a binary additive, CN\&DIO, afford a remarkable PCE of $13.58 \%$ with high $J_{\text {SC }}$ $\left(21.98 \mathrm{~mA} \mathrm{~cm}^{-2}\right)$ and $\mathrm{FF}(71.03 \%)$, which is among the highest efficiencies of OSCs. ${ }^{[6 \mathrm{c}-\mathrm{f}]}$

The synthetic route of IDCIC is shown in Scheme 1 and the synthetic details are presented in the Supporting Information. Compound 1, ethyl 2-bromothieno[3,2-b]thiophene-3-carboxylate, was prepared according to the literature. ${ }^{[14]}$ It was reacted with compound 2, (5,10-dihexylnaphtho[1,2-b:5,6-b) dithiophene2,7-diyl)bis(trimethylstannane), via Pd-catalyzed Stille coupling to afford the intermediate 3 . The addition of four equivalents of Grignard reagent to the ester groups in intermediate 3 followed by an acid-mediated intramolecular dehydration FriedelCrafts reaction produced the fused decacyclic donor core, IDC. IDC was then deprotonated by $n$-butyllithium at $-78{ }^{\circ} \mathrm{C}$ and subsequently quenched by dry $N, N$-dimethylformamide to give IDC-CHO. A Knoevenagel condensation between IDCCHO and 2-(5,6-difluoro-3-oxo-2,3-dihydro- $1 \mathrm{H}$-inden-1-ylidene) malononitrile afforded the final product IDCIC in $82 \%$ yield. The chemical structures of intermediates and IDCIC were confirmed by NMR and mass spectra, as shown in Figures S1-S3 in the Supporting Information. The thermal stability of IDCIC was determined by using thermogravimetric analysis, as shown in Figure S4 in the Supporting Information. The decomposition temperature $\left(T_{\mathrm{d}}, 5 \% \mathrm{wt}\right.$ loss) of IDCIC is $389^{\circ} \mathrm{C}$, indicating that it possesses splendid thermal stability.

The solution and thin-film absorption spectra of IDCIC were recorded on a UV-vis spectrophotometer and presented in Figure 1. The optical data are summarized in Table S1 in the Supporting Information. In solution, IDCIC exhibits a strong absorption in the region of $600-780 \mathrm{~nm}$ with a high maximum extinction coefficient of $3.3 \times 10^{5} \mathrm{M}^{-1} \mathrm{~cm}^{-1}$ at $719 \mathrm{~nm}$ (Figure S5, Supporting Information). From solution to thin film, the maximum absorption peak of IDCIC shows remarkable redshift from 719 to $766 \mathrm{~nm}$ with a broader absorption in $600-830 \mathrm{~nm}$, indicating the compact molecular stacking in the solid state of 


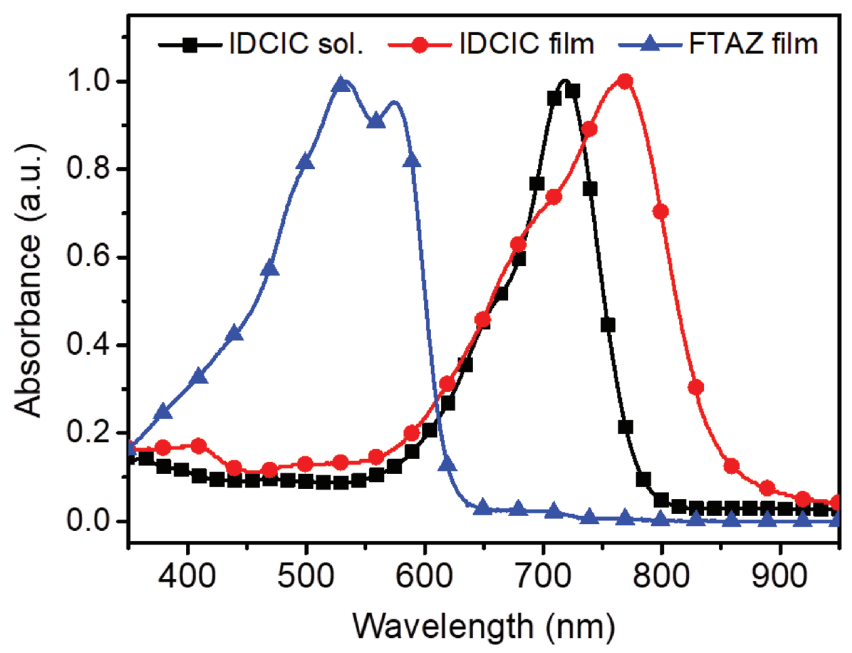

Figure 1. Absorption spectra of IDCIC in solution, and IDCIC and FTAZ as films.

IDCIC. According to the absorption onsets, the optical bandgap of IDCIC is as small as $1.45 \mathrm{eV}$. The high-performance wide bandgap polymer, FTAZ, ${ }^{[15]}$ was chosen as donor and it shows a strong absorption in the region of 400-600 nm, which complements well with IDCIC (Figure 1). The energy levels of IDCIC were measured by cyclic voltammetry (Figure S6, Supporting Information). The onset potentials of oxidation $\left(E_{\mathrm{ox}}{ }^{\circ}\right)$ and reduction $\left(E_{\text {red }}{ }^{\circ}\right)$ for IDCIC are 0.39 and $-1.08 \mathrm{~V}$ versus $\mathrm{Fc} / \mathrm{Fc}^{+}$, respectively. The highest occupied molecular orbital and lowest unoccupied molecular orbital energy levels of IDCIC were calculated to be -5.19 and $-3.72 \mathrm{eV}$, respectively. Thus, the electrochemical bandgap of IDCIC is $1.47 \mathrm{eV}$ and very close to its optical bandgap.

The inverted OSCs with a structure of ITO/ZnO/ FTAZ:IDCIC/ $\mathrm{MoO}_{3} / \mathrm{Ag}$ were fabricated to evaluate the photovoltaic performance of IDCIC. The photovoltaic data, $J-V$ curves and histograms of PCEs of the OSCs are collected in Table 1 and Figure 2, respectively. The FTAZ:IDCIC device with no additive affords a PCE of $9.64 \%$ with a $V_{\text {OC }}$ of $0.902 \mathrm{~V}$, a $J_{\text {SC }}$ of $17.45 \mathrm{~mA} \mathrm{~cm}^{-2}$, and an $\mathrm{FF}$ of $61.20 \%$. It has been confirmed that solvent additive can efficiently control the nanoscale morphology of the active layer during the film formation. ${ }^{[16]} 1,8$-Diiodooctane (DIO) and chloronaphthalene (CN) were chosen as additives in FTAZ:IDCIC system, which are widely used in OSCs. The FTAZ:IDCIC device affords a similar PCE of $9.76 \%$ after adding $0.25 \%$ DIO. However, the PCE of the FTAZ:IDCIC device with $0.25 \% \mathrm{CN}$ is enhanced to $11.81 \%$ with remarkably increased $J_{\mathrm{SC}}\left(20.95 \mathrm{~mA} \mathrm{~cm}^{-2}\right)$. It demonstrates that the selection of additive is very important to improve the photovoltaic performance of FTAZ:IDCIC-based OSCs. Furthermore, the
PCE of FTAZ:IDCIC based OSC is significantly enhanced to $13.58 \%$ with the highest $J_{\mathrm{SC}}\left(21.98 \mathrm{~mA} \mathrm{~cm}^{-2}\right)$ and $\mathrm{FF}(71.03 \%)$ by using a binary additive $0.25 \% \mathrm{CN} \& \mathrm{DIO}\left(V_{\mathrm{CN}}: V_{\mathrm{DIO}}=1: 1\right)$. The external quantum efficiency (EQE) curves of the FTAZ:IDCICbased OSCs without or with additive are presented in Figure $2 \mathrm{~b}$. The FTAZ:IDCIC-based OSC with $0.25 \%$ CN\&DIO gave the highest EQE with the maximum of $81.2 \%$ at $530 \mathrm{~nm}$. The corresponding integral current densities for FTAZ:IDCIC based OSCs with no additive, $0.25 \%$ DIO, $0.25 \% \mathrm{CN}$, and $0.25 \%$ CN\&DIO are 17.16, 16.87, 20.17, and $21.24 \mathrm{~mA} \mathrm{~cm}^{-2}$, respectively, which match well with the $J_{\mathrm{SC}}$ obtained from the $J-V$ measurements (the errors are all within 5\%).

The exciton/charge dynamics of the FTAZ:IDCIC-based devices without or with additive was investigated to explore the reason for the difference in their photovoltaic performance. The photoluminescence (PL) spectra of the FTAZ:IDCIC blend films with and without additive were measured to explore the charge separation behavior in the blends, as shown in Figure S7 in the Supporting Information. The selected excitation wavelength for FTAZ is $533 \mathrm{~nm}$ while that for IDCIC is $766 \mathrm{~nm}$ according to their maximum absorptions. When excited the donor FTAZ at a wavelength of $533 \mathrm{~nm}$, the PL spectrum of the FTAZ neat film appears in $575-775 \mathrm{~nm}$. For the blend films, their emissions are almost all quenched (by over 98\%), indicating effective electron transfer from FTAZ to IDCIC. For the acceptor IDCIC, its emission spectrum is located in the range of 780-870 nm when excited at a wavelength of $766 \mathrm{~nm}$. For the blend films, PL spectra are quenched by $83 \%$ for FTAZ:IDCIC with no additive, 77\% for FTAZ:IDCIC with $0.25 \%$ DIO, 90\% for FTAZ:IDCIC with $0.25 \% \mathrm{CN}$, and $92 \%$ for FTAZ:IDCIC with $0.25 \%$ CN\&DIO. Overall, the FTAZ:IDCIC-based device with $0.25 \%$ CN\&DIO exhibits the most efficient charge separation in the active layer. We studied monomolecular recombination in the FTAZ:IDCIC-based OSCs via treating their $V_{\mathrm{OC}}$ as a function of $P_{\text {light }} . V_{\mathrm{OC}}$ data were plotted against $\ln P_{\text {light }}$ (Figure 3a). ${ }^{[17]}$ The slopes of FTAZ:IDCIC based OSCs with no additive, $0.25 \%$ DIO, $0.25 \% \mathrm{CN}$, and $0.25 \% \mathrm{CN} \& \mathrm{DIO}$ are $1.37 \pm 0.05 \mathrm{kT} / \mathrm{q}, 1.56 \pm 0.06 \mathrm{kT} / \mathrm{q}, 1.15 \pm 0.03 \mathrm{kT} / \mathrm{q}$ and $1.20 \pm 0.03 \mathrm{kT} / \mathrm{q}$, respectively. The smaller slope indicates the less monomolecular recombination in the device. Thus, the addition of $0.25 \%$ DIO aggravates the monomolecular recombination, while the addition of $0.25 \% \mathrm{CN}$ reduces it efficiently in the FTAZ:IDCIC based-OSCs. Their $J_{\text {SC }}$ as a function of incident light intensity, $P_{\text {light }}$, was also studied to explore bimolecular recombination in the active layers. The data were fitted to the power law: $J_{\mathrm{SC}} \propto P_{\text {light }}{ }^{\alpha}$ (Figure 3b). ${ }^{[18]} \alpha$ for the FTAZ:IDCICbased OSCs with no additive, $0.25 \%$ DIO, $0.25 \% \mathrm{CN}$, and $0.25 \%$ CN\&DIO are $0.952 \pm 0.008,0.999 \pm 0.006,0.918 \pm 0.012$, and $0.999 \pm 0.009$, respectively. As $\alpha$ is closer to 1 , it implies the

Table 1. Photovoltaic parameters of FTAZ:IDCIC solar cells without or with additive under AM $1.5 \mathrm{G}$ illumination at $100 \mathrm{~mW} \mathrm{~cm}$. The average values and standard deviations of 20 devices are shown in parentheses.

\begin{tabular}{lccccc}
\hline & $V_{\mathrm{OC}}[\mathrm{V}]$ & $J_{S C}\left[\mathrm{~mA} \mathrm{~cm}^{-2}\right]$ & $\mathrm{FF}[\%]$ & $\mathrm{PCE}[\%]$ & $J_{\mathrm{EQE}}\left[\mathrm{mA} \mathrm{cm}{ }^{-2}\right]$ \\
\hline No additive & $0.902(0.917 \pm 0.010)$ & $17.45(17.27 \pm 0.44)$ & $61.20(57.41 \pm 1.79)$ & $9.64(9.09 \pm 0.34)$ & 17.16 \\
$0.25 \%$ DIO & $0.930(0.925 \pm 0.005)$ & $17.17(17.49 \pm 0.33)$ & $61.13(59.14 \pm 1.43)$ & $9.76(9.56 \pm 0.14)$ & 16.87 \\
$0.25 \%$ CN & $0.864(0.860 \pm 0.005)$ & $20.95(21.25 \pm 0.33)$ & $65.24(63.12 \pm 1.12)$ & $11.81(11.55 \pm 0.19)$ & 20.17 \\
$0.25 \%$ CN\&DIO & $0.870(0.865 \pm 0.003)$ & $21.98(21.77 \pm 0.18)$ & $71.03(69.58 \pm 0.80)$ & $13.58(13.10 \pm 0.26)$ & 21.24 \\
\hline
\end{tabular}


(a)

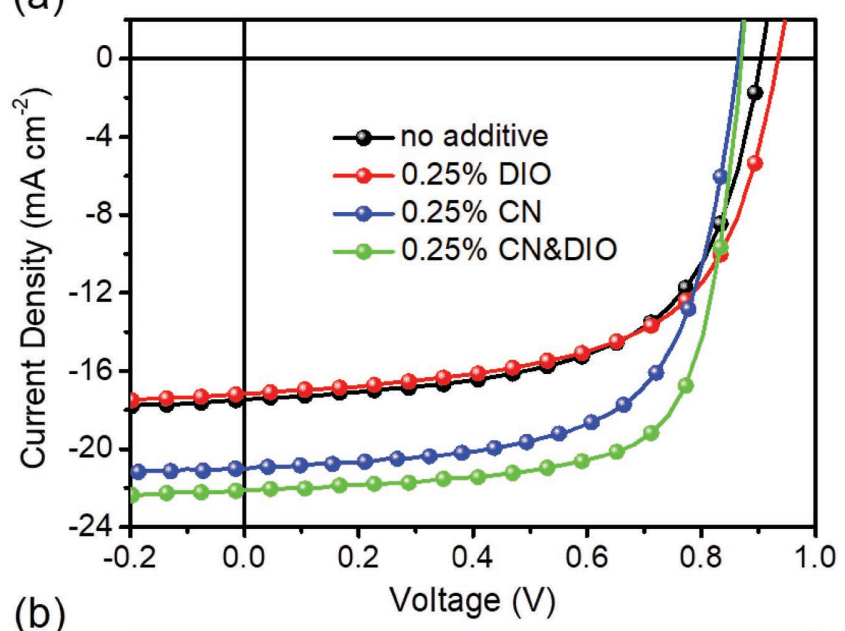

(b)

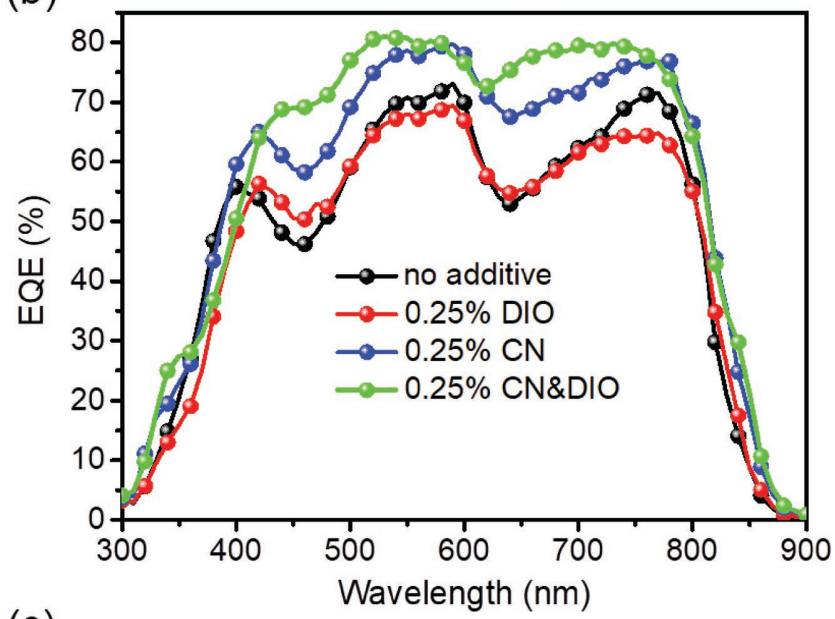

(c)
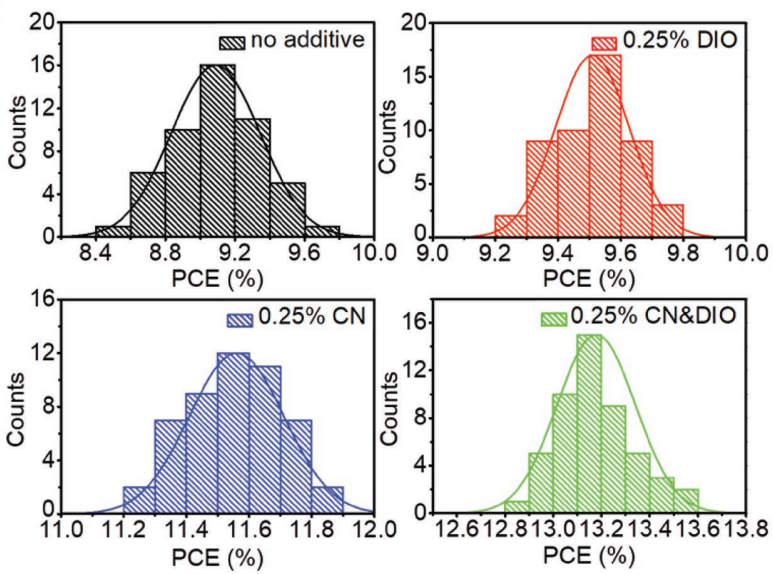

Figure 2. a) $J-V$ curves, b) EQE spectra, and c) histograms of PCEs of FTAZ:IDCIC-based OSCs without or with additive.

less bimolecular recombination in the device. Thus, the addition of $0.25 \%$ DIO inhibits bimolecular recombination, while the addition of $0.25 \% \mathrm{CN}$ deteriorates it in the FTAZ:IDCICbased OSCs. Furthermore, space charge limited current method was used to determine charge carrier mobilities of the blend films (Figure S8, Supporting Information). The electron mobilities of the FTAZ:IDCIC blend films with no additive, (a)

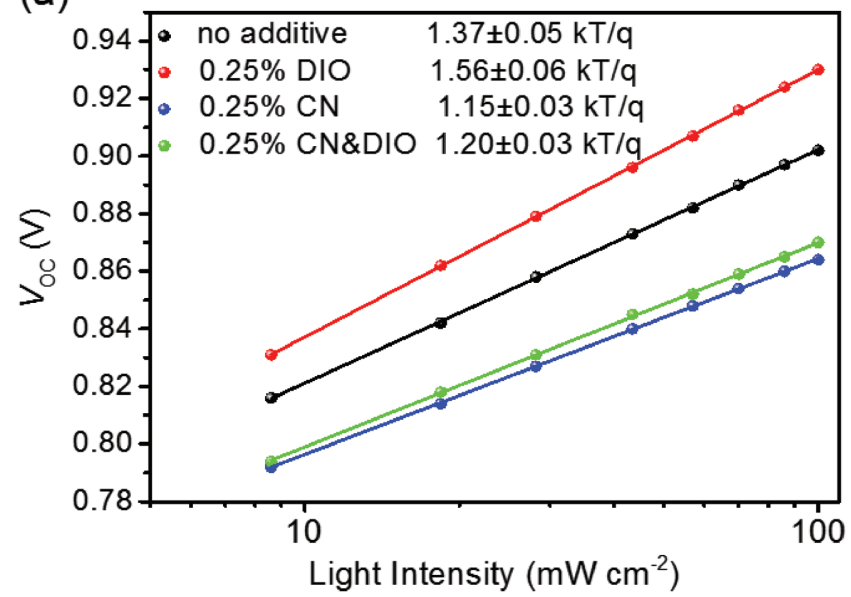

(b)

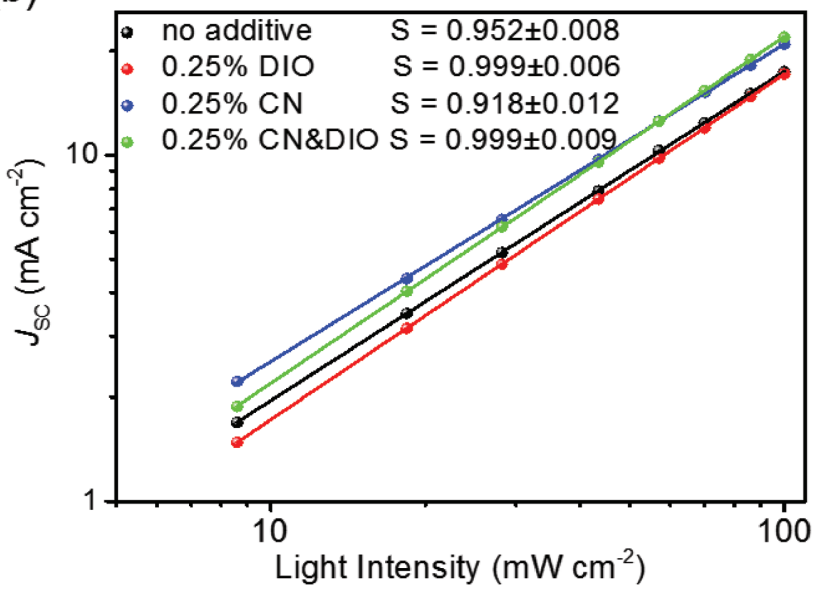

Figure 3. a) Dependence of $V_{\mathrm{OC}}$ and b) dependence of $J_{\mathrm{SC}}$ on light intensity for FTAZ:IDCIC-based OSCs without or with additive.

$0.25 \%$ DIO, $0.25 \% \mathrm{CN}$ and $0.25 \% \mathrm{CN} \& \mathrm{DIO}$ are $6.5 \times 10^{-5}$, $7.7 \times 10^{-5}, 1.3 \times 10^{-4}$, and $1.5 \times 10^{-4} \mathrm{~cm}^{2} \mathrm{~V}^{-1} \mathrm{~s}^{-1}$, respectively, while the hole mobilities of the blend films with no additive, $0.25 \% \mathrm{DIO}, 0.25 \% \mathrm{CN}$, and $0.25 \% \mathrm{CN} \& \mathrm{DIO}$ are $2.1 \times 10^{-4}$, $2.4 \times 10^{-4}, 2.8 \times 10^{-4}$, and $3.0 \times 10^{-4} \mathrm{~cm}^{2} \mathrm{~V}^{-1} \mathrm{~s}^{-1}$, respectively. Thus, the $\mu_{\mathrm{h}} / \mu_{\mathrm{e}}$ of the FTAZ:IDCIC blend films with no additive, $0.25 \% \mathrm{DIO}, 0.25 \% \mathrm{CN}$, and $0.25 \% \mathrm{CN} \& \mathrm{DIO}$ are $3.2,3.1$, 2.2, and 2.0, respectively. The highest and most balanced charge carrier mobilities for the blend film with $0.25 \%$ CN\&DIO benefit charge transport and high FF in the OSC. Based on the results above, it can be found that the binary additive CN\&DIO combines the advantage of DIO and $\mathrm{CN}$ together. As a result, the FTAZ:IDCIC-based device with $0.25 \%$ CN\&DIO exhibits the most efficient charge separation, least charge recombination and highest charge carrier mobilities, thus contributing to its highest $J_{\mathrm{SC}}$ and FF in the OSC.

The morphology of the active layers based on FTAZ:IDCIC without or with additive was studied by atomic force microscopy (AFM), grazing-incidence wide-angle X-ray scattering (GIWAXS) and resonant soft X-ray scattering (R-SoXS). In the AFM height images (Figure S9, Supporting Information), the root-mean-square (RMS) roughness of FTAZ:IDCIC blend 

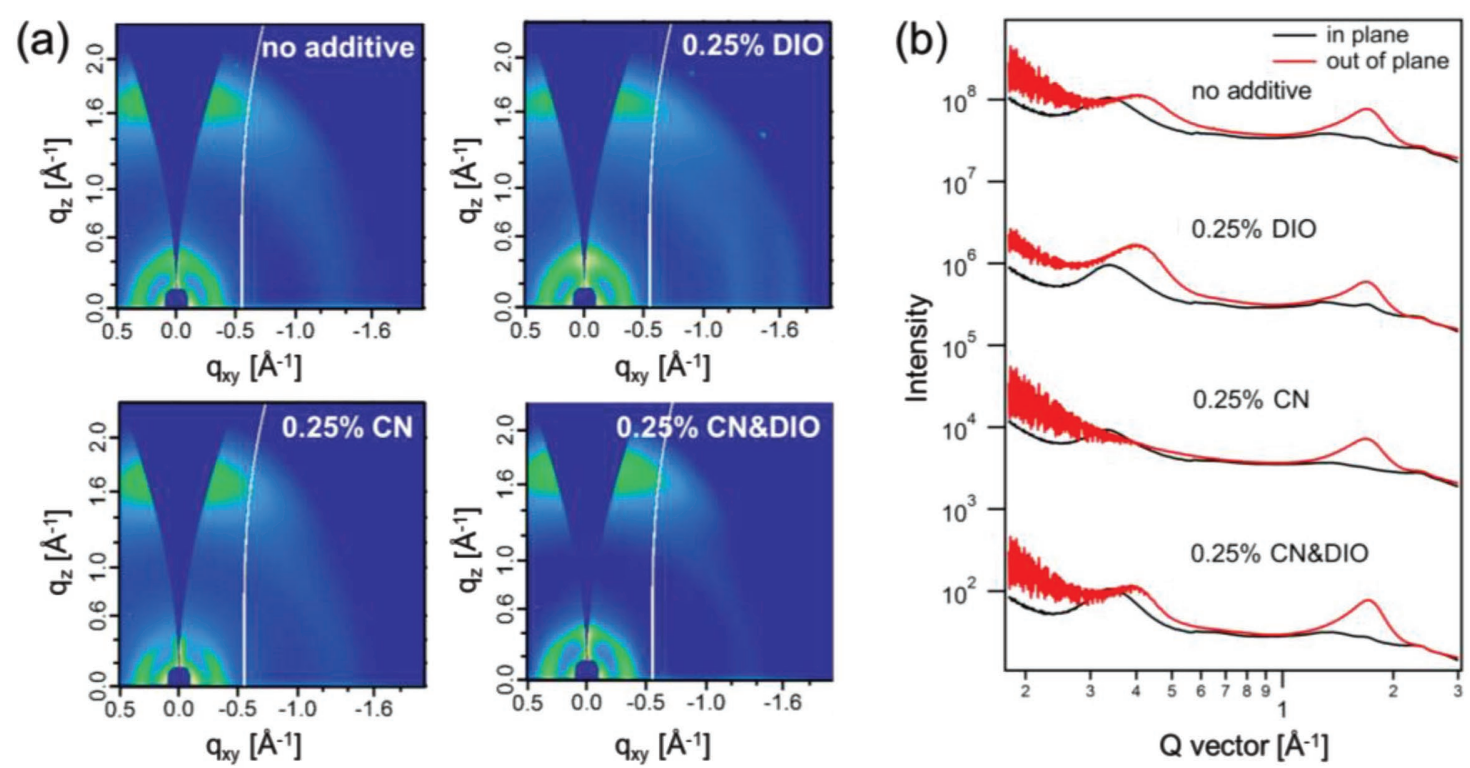

Figure 4. a) 2D GIWAXS patterns for FTAZ:IDCIC blend films without or with additive. b) Corresponding GIWAXS intensity profiles along the in-plane and out-of-plane directions.

films with no additive, $0.25 \%$ DIO, $0.25 \% \mathrm{CN}$, and $0.25 \%$ CN\&DIO are $1.15,1.84,1.06$, and $1.08 \mathrm{~nm}$, respectively. As shown in the phase images (Figure S9, Supporting Information), there is no obvious phase separation in the FTAZ:IDCIC blend film with no additive while the FTAZ:IDCIC blend film shows large aggregations after adding 0.25\% DIO. However, the FTAZ:IDCIC blend film with $0.25 \%$ CN exhibits clear phase separation with nanostructure, which increases the D/A interface and benefits exciton dissociation for the improved $J_{\mathrm{SC}}$. Furthermore, after adding 0.25\% CN\&DIO, the FTAZ:IDCIC blend film has more subtle morphology with clearer phase separation, which contributes to the least charge recombination and highest $J_{\mathrm{SC}}$ and FF in the OSC. GIWAXS was conducted to investigate the molecular orientation and packing in the blend films. ${ }^{[19]}$ All blend films prefer to face-on orientation relative to the substrates (Figure $4 \mathrm{a}$ ). The $\pi-\pi$ stacking peaks in the out-of-plane direction of FTAZ:IDCIC blend films without or with additive are all located at $\approx 1.70 \AA^{-1}$, corresponding to the $\pi-\pi$ stacking $\mathrm{d}$-spacing of $\approx 3.69 \AA$ (Figure $4 \mathrm{~b}$ ). The peaks located at $\approx 0.32 \AA^{-1}$ in the in-plane direction are assigned to the (100) lamellar stacking scattering of FTAZ, corresponding to the lamellar stacking $d$-spacing of $\approx 19.36 \AA$, which is consistent with the neat FTAZ film in Figure S10 in the Supporting Information. The peaks located at $\approx 0.39 \AA^{-1}$ in the out-of-plane direction are attributable to the (100) lamellar stacking scattering of IDCIC, corresponding to the lamellar stacking d-spacing of $\approx 15.94 \AA$, as the neat IDCIC film (Figure S10, Supporting Information). The lamellar stacking scattering peaks of IDCIC and FTAZ heighten for the blend film after adding 0.25\% DIO, while both $\pi-\pi$ and lamellar stacking scattering peaks descend for the blend film with $0.25 \%$ CN. For the FTAZ:IDCIC blend film with $0.25 \% \mathrm{CN} \& \mathrm{DIO}$, the intensities of $\pi-\pi$ and lamellar stacking scattering peaks both increase. The coherence length (CL) of $\pi-\pi$ stacking in the FTAZ:IDCIC blend films also shows the same trend. By adding $0.25 \% \mathrm{CN}$, the CL of FTAZ:IDCIC blend film remains at $1.61 \mathrm{~nm}$, while a clear increment of CL (1.74 nm) can be observed in the blend film with $0.25 \%$ DIO. And the binary additive $(0.25 \%$ CN\&DIO) further improves the CL $(1.78 \mathrm{~nm})$, which indicates that the molecular stacking gets more ordered, thus benefiting charge transport in the OSC. R-SoXS was employed to obtain the phase separation information of the small molecule acceptors based blend films (Figure 5). In order to achieve an optimal material contrast, a series of photon energies were employed to measure the R-SoXS from 270 to $286.8 \mathrm{eV}$. For soft X-ray with different photon energy, each material shows different absorption ability according to the near edge X-ray absorption fine structure. At the absorption edge, an extreme spectra leap occurs and creates the maximal contrast for two materials. Herein, the photon

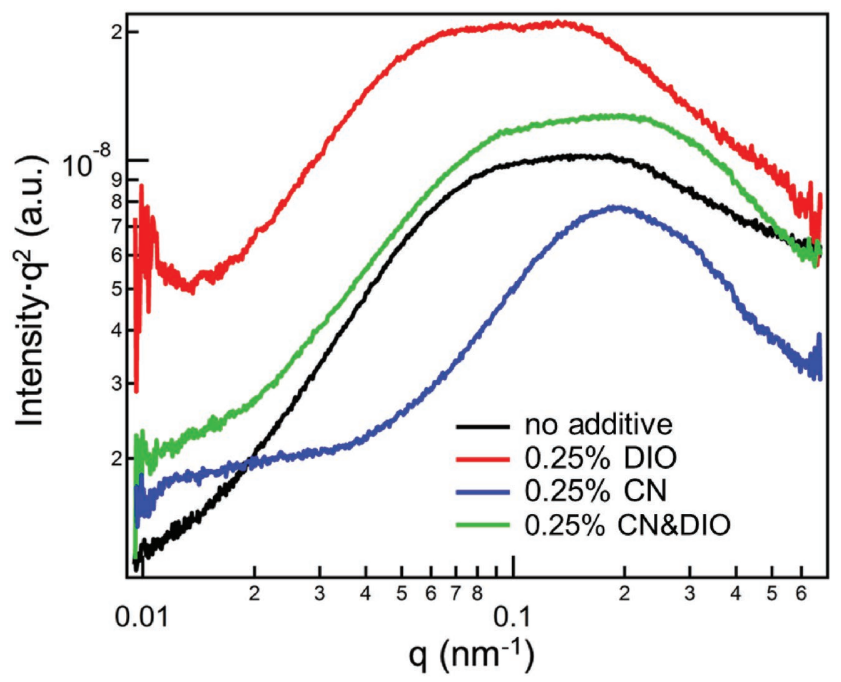

Figure 5. R-SoXS profiles in log scale for FTAZ:IDCIC blend films without or with additive. 
energy of $284.8 \mathrm{eV}$ was selected to detect the phase separation and present the best result. ${ }^{[20]}$ The FTAZ:IDCIC blend films with no additive, $0.25 \%$ DIO, and $0.25 \%$ CN\&DIO show hierarchical morphology feature with phase separation domain sizes of $34.49 / 11.49 \mathrm{~nm}, 62.97 / 26.06 \mathrm{~nm}$, and 33.58/11.49 nm, respectively, while the blend film with $0.25 \% \mathrm{CN}$ exhibits mono length scale at $0.39 \mathrm{~nm}^{-1}$, corresponding to the domain size of $16.10 \mathrm{~nm}$. It is possible that the phase separation at the larger length scales is caused by the preaggregation of IDCIC in the solution. And the relative domain purities in the FTAZ:IDCIC blend films with no additive, $0.25 \%$ DIO, $0.25 \% \mathrm{CN}$, and $0.25 \%$ CN\&DIO were calculated to be $0.90,1.16,0.73$, and 1 , respectively. The big difference in morphology and photovoltaic performance should be ascribed to the effect of the additives. The determined solubilities of IDCIC in chloroform, DIO, and CN at room temperature are $12.5,<0.1$, and $23.3 \mathrm{mg} \mathrm{mL}^{-1}$, respectively. The poor solubility in DIO for IDCIC promotes it to aggregate and enhances the domain size and domain purity, thus reducing bimolecular recombination but leading to the more severe monomolecular recombination. On the contrary, the excellent solubility in $\mathrm{CN}$ for IDCIC inhibits the aggregation, interferes molecular packing, and reduces the domain size and domain purity, thus affording more D/A interfaces and benefiting exciton dissociation but aggravating bimolecular recombination. The binary additive, CN\&DIO, compromises the effect of the two above. As a result, the FTAZ:IDCIC blend film with $0.25 \%$ CN\&DIO obtains the best morphology with proper domain size and high domain purity simultaneously, thus achieving the highest $J_{\mathrm{SC}}$ and FF in the OSC.

In summary, a new fused decacyclic unit via fusing naphtho[1,2-b:5,6- $b$ ' dithiophene with two thieno[3,2- $b]$ thiophenes was developed and used as the donor core to prepare the FREA, IDCIC. IDCIC exhibits broad and strong absorption in the region of $600-830 \mathrm{~nm}$ with a small optical bandgap of $1.45 \mathrm{eV}$. The FTAZ:IDCIC-based OSCs afford an impressive PCE of $13.58 \%$ with high $J_{\mathrm{SC}}\left(21.98 \mathrm{~mA} \mathrm{~cm}^{-2}\right)$ and FF $(71.03 \%)$ by using the binary additive, CN\&DIO. To the best of our knowledge, the PCE of this work is the highest efficiency for FREA with such large fused ring donor core. It demonstrates that extending the $\pi$-conjugation of the donor core is an efficient method to achieve high PCE for FREAs. In addition, the solubility of IDCIC in additive plays an important role in controlling the morphology of active layer based on FTAZ:IDCIC, thus the photovoltaic performance. The poor solubility in DIO for IDCIC promotes it to aggregate and enhances the domain size and domain purity in the FTAZ:IDCIC blend film, while the excellent solubility in $\mathrm{CN}$ for IDCIC inhibits the aggregation, interferes molecular packing, facilitates it to diffuse into the donor FTAZ, and reduces the domain size and domain purity. The binary additive, CN\&DIO, compromises the effect of the two, thus the FTAZ:IDCIC-based OSC with $0.25 \%$ CN\&DIO gets the best morphology with moderate domain size and high domain purity simultaneously, achieving the most efficient charge separation, least charge recombination, and highest charge carrier mobilities for the highest $J_{\mathrm{SC}}$ and FF. Therefore, combining good and poor solvent as the binary additive is an alternatively effective strategy to optimize the morphology of active layer for high PCE. Further study on FREAs with large fused ring donor core and the effect of additive on the morphology is ongoing.

\section{Supporting Information}

Supporting Information is available from the Wiley Online Library or from the author.

\section{Acknowledgements}

The authors thank the National Postdoctoral Program for Innovative Talents (Grant No. BX201700253) and the China Postdoctoral Science Foundation (Grant Nos. 2017M620068, 2018M630208). The authors also thank the National Natural Science Foundation of China (Grant Nos. 21673257, 21504066). The authors thank for the support from Ministry of Science and Technology (No. 2016YFA0200700) for their support. X-ray data were acquired at beamlines 7.3.3 and 11.0.1.2 at the Advanced Light Source, which is supported by the Director, Office of Science, Office of Basic Energy Sciences, of the U.S. Department of Energy under Contract No. DE-AC02-05CH11231. The authors thank Chenhui Zhu at beamline 7.3.3 and Cheng Wang at beamline 11.0.1.2 for assistance with data acquisition.

\section{Conflict of Interest}

The authors declare no conflict of interest.

\section{Keywords}

additive optimization, fused decacyclic unit, fused ring electron acceptor, organic solar cells, power conversion efficiency

Received: July 3, 2018

Revised: August 6, 2018

Published online: September 14, 2018

[1] a) V. Shrotriya, Nat. Photonics 2009, 3, 447; b) R. Søndergaard, M. Hösel, D. Angmo, T. T. Larsen-Olsen, F. C. Krebs, Mater. Today 2012, 15, 36.

[2] a) Y.-J. Cheng, S.-H. Yang, C.-S. Hsu, Chem. Rev. 2009, 109, 5868; b) O. Ostroverkhova, Chem. Rev. 2016, 116, 13279; c) P. Cheng, G. Li, X. Zhan, Y. Yang, Nat. Photonics 2018, 12, 131.

[3] a) J.-S. Wu, S.-W. Cheng, Y.-J. Cheng, C.-S. Hsu, Chem. Soc. Rev. 2015, 44, 1113; b) C.-Z. Li, H.-L. Yip, A. K.-Y. Jen, J. Mater. Chem. 2012, 22, 4161; c) C. Yan, S. Barlow, Z. Wang, H. Yan, A. K.-Y. Jen, S. R. Marder, X. Zhan, Nat. Rev. Mater. 2018, 3, 18003; d) H.-Y. Chen, J. Hou, S. Zhang, Y. Liang, G. Yang, Y. Yang, L. Yu, Y. Wu, G. Li, Nat. Photonics 2009, 3, 649; e) J. Zhao, Y. Li, G. Yang, K. Jiang, H. Lin, H. Ade, W. Ma, H. Yan, Nat. Energy 2016, 1, 15027; f) M. An, F. Xie, X. Geng, J. Zhang, J. Jiang, Z. Lei, D. He, Z. Xiao, L. Ding, Adv. Energy Mater. 2017, 7, 1602509; g) H. Li, D. He, P. Mao, Y. Wei, L. Ding, J. Wang, Adv. Energy Mater. 2017, 7, 1602663; h) F. Zhao, X. Meng, Y. Feng, Z. Jin, Q. Zhou, H. Li, L. Jiang, J. Wang, Y. Li, C. Wang, J. Mater. Chem. A 2015, 3, 14991; i) Z. Xiao, X. Geng, D. He, X. Jia, L. Ding, Energy Environ. Sci. 2016, 9, 2114.

[4] a) Y. Lin, X. Zhan, Mater. Horiz. 2014, 1, 470; b) Y. Lin, X. Zhan, Adv. Energy Mater. 2015, 5, 1501063; c) Z. Liu, Y. Wu, Q. Zhang, X. Gao, J. Mater. Chem. A 2016, 4, 17604. 
[5] a) D. He, F. Zhao, L. Jiang, C. Wang, J. Mater. Chem. A 2018, 6, 8839 ; b) Y. Liu, C. Zhang, D. Hao, Z. Zhang, L. Wu, M. Li, S. Feng, X. Xu, F. Liu, X. Chen, Z. Bo, Chem. Mater. 2018, 30, 4307.

[6] a) Y. Lin, J. Wang, Z.-G. Zhang, H. Bai, Y. Li, D. Zhu, X. Zhan, Adv. Mater. 2015, 27, 1170; b) F. Zhao, S. Dai, Y. Wu, Q. Zhang, J. Wang, L. Jiang, Q. Ling, Z. Wei, W. Ma, W. You, C. Wang, X. Zhan, Adv. Mater. 2017, 29, 1700144; c) W. Zhao, S. Li, H. Yao, S. Zhang, Y. Zhang, B. Yang, J. Hou, J. Am. Chem. Soc. 2017, 139, 7148; d) Z. Fei, F. D. Eisner, X. Jiao, M. Azzouzi, J. A. Röhr, Y. Han, M. Shahid, A. S. R. Chesman, C. D. Easton, C. R. McNeill, T. D. Anthopoulos, J. Nelson, M. Heeney, Adv. Mater. 2018, 30, 1705209; e) Z. Xiao, X. Jia, L. Ding, Sci. Bull. 2017, 62, 1562; f) S. Zhang, Y. Qin, J. Zhu, J. Hou, Adv. Mater. 2018, 30, 1800868.

[7] a) K. N. Winzenberg, P. Kemppinen, F. H. Scholes, G. E. Collis, Y. Shu, T. B. Singh, A. Bilic, C. M. Forsyth, S. E. Watkins, Chem. Commun. 2013, 49, 6307; b) Y. Kim, C. E. Song, S.-J. Moonc, E. Lim, Chem. Commun. 2014, 50, 8235; c) S. Holliday, R. S. Ashraf, C. B. Nielsen, M. Kirkus, J. A. Röhr, C.-H. Tan, E. Collado-Fregoso, A.-C. Knall, J. R. Durrant, J. Nelson, I. McCulloch, J. Am. Chem. Soc. 2015, 137, 898; d) K. Wang, Y. Firdaus, M. Babics, F. Cruciani, Q. Saleem, A. E. Labban, M. A. Alamoudi, T. Marszalek, W. Pisula, F. Laquai, P. M. Beaujuge, Chem. Mater. 2016, 28, 2200.

[8] a) Y. Lin, Z.-G. Zhang, H. Bai, J. Wang, Y. Yao, Y. Li, D. Zhu, X. Zhan, Energy Environ. Sci. 2015, 8, 610; b) S. Feng, C. Zhang, Y. Liu, Z. Bi, Z. Zhang, X. Xu, W. Ma, Z. Bo, Adv. Mater. 2017, 29, 1703527; c) Y. Lin, Q. He, F. Zhao, L. Huo, J. Mai, X. Lu, C.-J. Su, T. Li, J. Wang, J. Zhu, Y. Sun, C. Wang, X. Zhan, J. Am. Chem. Soc. 2016, 138, 2973; d) S. Holliday, R. S. Ashraf, A. Wadsworth, D. Baran, S. A. Yousaf, C. B. Nielsen, C.-H. Tan, S. D. Dimitrov, Z. Shang, N. Gasparini, M. Alamoudi, F. Laquai, C. J. Brabec, A. Salleo, J. R. Durrant, I. McCulloch, Nat. Commun. 2016, 7, 11585.

[9] a) Y.-Q.-Q. Yi, H. Feng, M. Chang, H. Zhang, X. Wan, C. Li, Y. Chen, J. Mater. Chem. A 2017, 5, 17204; b) Y. Ma, M. Zhang, Y. Yan, J. Xin, T. Wang, W. Ma, C. Tang, Q. Zheng, Chem. Mater. 2017, 29, 7942; c) J. Zhang, C. Yan, W. Wang, Y. Xiao, X. Lu, S. Barlow, T. C. Parker, X. Zhan, S. R. Marder, Chem. Mater. 2018, 30, 309.
[10] a) Y. Lin, F. Zhao, Q. He, L. Huo, Y. Wu, T. C. Parker, W. Ma, Y. Sun, C. Wang, D. Zhu, A. J. Heeger, S. R. Marder, X. Zhan, J. Am. Chem. Soc. 2016, 138, 4955; b) S. Li, L. Ye, W. Zhao, S. Zhang, S. Mukherjee, H. Ade, J. Hou, Adv. Mater. 2016, 28, 9423; c) H. Yao, L. Ye, J. Hou, B. Jang, G. Han, Y. Cui, G. M. Su, C. Wang, B. Gao, R. Yu, H. Zhang, Y. Yi, H. Y. Woo, H. Ade, J. Hou, Adv. Mater. 2017, 29, 1700254.

[11] a) B. Kan, H. Feng, X. Wan, F. Liu, X. Ke, Y. Wang, Y. Wang, H. Zhang, C. Li, J. Hou, Y. Chen, J. Am. Chem. Soc. 2017, 139, 4929; b) J. Wang, W. Wang, X. Wang, Y. Wu, Q. Zhang, C. Yan, W. Ma, W. You, X. Zhan, Adv. Mater. 2017, 29, 1702125; c) Y. Li, L. Zhong, B. Gautam, H.-J. Bin, J.-D. Lin, F.-P. Wu, Z. Zhang, Z.-Q. Jiang, Z.-G. Zhang, K. Gundogdu, Y. Li, L.-S. Liao, Energy Environ. Sci. 2017, 10, 1610.

[12] a) J. Zhu, Z. Ke, Q. Zhang, J. Wang, S. Dai, Y. Wu, Y. Xu, Y. Lin, W. Ma, W. You, X. Zhan, Adv. Mater. 2018, 30, 1704713; b) T. Li, S. Dai, Z. Ke, L. Yang, J. Wang, C. Yan, W. Ma, X. Zhan, Adv. Mater. 2018, 30, 1705969.

[13] a) Y. Li, X. Liu, F.-P. Wu, Y. Zhou, Z.-Q. Jiang, B. Song, Y. Xia, Z.-G. Zhang, F. Gao, O. Inganäs, Y. Li, L.-S. Liao, J. Mater. Chem. A 2016, 4, 5890; b) B. Jia, S. Dai, Z. Ke, C. Yan, W. Ma, X. Zhan, Chem. Mater. 2018, 30, 239.

[14] D. He, L. Qian, L. Ding, Polym. Chem. 2016, 7, 2329.

[15] S. C. Price, A. C. Stuart, L. Yang, H. Zhou, W. You, J. Am. Chem. Soc. 2011, 133, 4625.

[16] F. Zhao, C. Wang, X. Zhan, Adv. Energy Mater. 2018, 8, 1703147.

[17] a) S. R. Cowan, A. Roy, A. J. Heeger, Phys. Rev. B 2010, 82, 245207; b) F. Xie, D. He, H. Pan, J. Jiang, L. Ding, Macromol. Rapid Commun. 2017, 38, 1700074.

[18] a) F. Zhao, Z. Wang, J. Zhang, X. Zhu, Y. Zhang, J. Fang, D. Deng, Z. Wei, Y. Li, L. Jiang, C. Wang, Adv. Energy Mater. 2016, 6, 1502120; b) D. He, X. Geng, L. Ding, Polym. Chem. 2016, 7, 4993.

[19] A. Hexemer, W. Bras, J. Glossinger, E. Schaible, E. Gann, R. Kirian, A. MacDowell, M. Church, B. Rude, H. Padmore, J. Phys. Conf. Ser. 2010, 247, 012007.

[20] a) E. Gann, A. T. Young, B. A. Collins, H. Yan, J. Nasiatka, H. A. Padmore, H. Ade, A. Hexemer, C. Wang, Rev. Sci. Instrum. 2012, 83, 045110; b) Y. Wu, Z. Wang, X. Meng, W. Ma, Prog. Chem. 2017, 29, 93. 